\title{
List of contributing authors
}

\section{Dr. Karl-Anders Weiß}

Fraunhofer Institute for

Solar Energy Systems ISE

Energy Technologies and Systems

Freiburg, Germany

karl-anders.weiss@ise.fraunhofer.de

\section{Sina Herceg}

Fraunhofer Institute for

Solar Energy Systems ISE

Service Life Analysis and Material

Characterization

Freiburg, Germany

sina.herceg@ise.fraunhofer.de

\section{Dr. Bengt Jäckel}

Fraunhofer Center for Silicon

Photovoltaics (CSP)

Module and System Reliability

Halle, Germany

bengt.jaeckel@csp.fraunhofer.de

\section{Dr. Ismail Kaaya}

Fraunhofer Institute for

Solar Energy Systems ISE

Service Life Analysis and Material

Characterization

Freiburg, Germany

ismail.kaaya@ise.fraunhofer.de

\section{Elisabeth Klimm}

EK Reliability

Wittnau, Germany

e.klimm@ek-reliability.de

\section{Dr. Gernot Oreški}

Polymer Competence Center Leoben $\mathrm{GmbH}$

Smart Material and Surface Testing

Leoben, Austria

gernot.oreski@pccl.at

\section{Sebastián Pinto Bautista}

Helmholtz-Institut HIU Elektrochemische

Energiespeicherung

Technology Assessment and System Analysis

Karlsruhe, Germany

sebastian.bautista@kit.edu

\section{Djamel Eddine Mansour}

Fraunhofer Institute for Solar Energy

Systems (ISE)

Freiburg, Germany

djamel.eddine.mansour@ise.fraunhofer.de 
